\title{
Itinerant electron metamagnetism and magnetocaloric effect in $\mathrm{Dy}(\mathrm{Co}, \mathrm{Si})_{2}$
}

\author{
Niraj K. Singh ${ }^{\mathrm{a}, *}$, K.G. Suresh ${ }^{\mathrm{a}}$, A.K. Nigam ${ }^{\mathrm{b}}$ \\ ${ }^{a}$ Department of Physics, Indian Institute of Technology Bombay, Mumbai 400 076, India \\ ${ }^{\mathrm{b}}$ Tata Institute of Fundamental Research, Homi Bhabha Road, Mumbai 400 005, India
}

\begin{abstract}
Itinerant electron metamagnetism in $\mathrm{Dy}\left(\mathrm{Co}_{1-x} \mathrm{Si}_{x}\right)_{2}$ compounds was studied in the light of a recent theoretical model based on magnetovolume effect and spin fluctuations. The nature of the magnetic transition in these compounds was analyzed within the framework of this model. The magnetocaloric effect in these compounds has been calculated and correlated with the strength of itinerant electron metamagnetism. The domain wall pinning effect was found to be dominant at low temperatures.
\end{abstract}

PACS: $75.30 . \mathrm{Sg} ; 75.30 . \mathrm{Kz}$

Keywords: A. Magnetically ordered materials; D. Thermodynamic properties

\section{Introduction}

Rare earth (R) transition metal (TM) intermetallics show interesting magnetic properties, which make them suitable candidates for fundamental studies as well as for applications [1,2]. Compounds exhibiting first order transitions (FOT) are potential candidates for applications based on magnetocaloric effect (MCE) and magnetoresistance (MR) [2]. Among the various $\mathrm{R}-\mathrm{TM}$ intermetallics, $\mathrm{RCo}_{2}$ $(\mathrm{R}=\mathrm{Dy}$, Ho and Er) compounds have received considerable attention owing to the presence of FOT at their ordering temperatures $\left(T_{\mathrm{C}}\right)[3]$. It has been found that the FOT in these compounds is due to the onset of itinerant electron metamagnetism (IEM), caused by the exchange field of the rare earth [4]. According to the earlier theories of IEM, the critical parameter that determines the possibility of IEM and thereby the order of transition is the molecular field or the ordering temperature. However, the second order transitions (SOT) observed in $\mathrm{RCo}_{2}$ compounds containing rare earths other than Ho, Dy and Er could not be satisfactorily explained using these theories, though they predicted FOT in $\mathrm{DyCo}_{2}, \mathrm{HoCo}_{2}$ and $\mathrm{ErCo}_{2}$ [5]. Recently, these models have been corrected by incorporating the spin fluctuation and magnetovoulme effects [5-7]. These refined models have been found to be in good agreement with the experimental results in many compounds [6,7]. In the case of $\mathrm{RCo}_{2}$ compounds, the model proposed by Khmelevskyi and Mohn [5] appears to be the unified model to describe IEM and the order of transition for both light and heavy rare earths.

In this paper, we report our results on the magnetization and MCE in $\mathrm{Dy}\left(\mathrm{Co}_{1-x} \mathrm{Si}_{x}\right)_{2}(x=0,0.075$ and 0.15$)$ compounds. Since $\mathrm{DyCo}_{2}$ is a classical IEM system which shows considerable MCE, it is of interest to study IEM and MCE as a function of $\mathrm{Si}$ concentration. We try to correlate the variations in the order of transition and MCE in these compounds, within the purview of Khmelevskyi and Mohn model. 


\section{Experimental details}

All the compounds were prepared by arc melting stoichiometric amounts of the constituent elements under argon atmosphere and subsequently annealing the ingots at $950{ }^{\circ} \mathrm{C}$ for a week. The samples were characterized by using powder X-ray diffractograms. The magnetization $(M)$ was measured in the temperature range $2-300 \mathrm{~K}$ using a Quantum Design SQUID magnetometer/OXFORD vibrating sample magnetometer. The temperature variation of magnetization was carried out both under zero-field cooled (ZFC) and field-cooled (FC) modes. MCE was calculated from the $M$ vs. $H$ plots at various temperatures close to $T_{\mathrm{C}}$.

\section{Results and discussion}

Powder X-ray diffraction patterns taken at room temperature revealed that all the compounds have formed in single phase with $\mathrm{C} 15$ Cubic Laves phase $\mathrm{MgCu}_{2}$ type structure. Fig. 1 shows the temperature variation of magnetization at 500 Oe under ZFC condition. The ordering temperatures have been calculated from these plots. The variation of lattice parameter, Curie temperature and saturation magnetization (at $2 \mathrm{~K}$ ) are given in Table 1 .

The variation of $T_{\mathrm{C}}$ with $\mathrm{Si}$ concentration in this system is similar to that in $\mathrm{ErCo}_{2}$ [8]. Substitution of Si gives rise to magnetovolume effect, chemical effect and magnetic dilution effect. The initial increase in $T_{\mathrm{C}}$ may be due to the predominant magnetovolume effect as a result of the $3 \mathrm{~d}$ band narrowing following lattice expansion. The subsequent decrease in $T_{\mathrm{C}}$ indicates that there are competing effects from the magnetic dilution and charge transfer between the $3 \mathrm{~d}$ band of Co and Si. The Co moments $\left(\mu_{3 \mathrm{~d}}\right)$ have been calculated by taking Dy moments to be $9 \mu_{B}$ and also by assuming antiferromagnetic coupling between Dy and Co moments. It can be seen from Table 1 that there is an increase in $\mu_{3 \mathrm{~d}}$ with $\mathrm{Si}$ concentration, which reflects the magnetovolume effect.

It can be seen from Fig. 1 that the magnetization changes abruptly at $T_{\mathrm{C}}$ in $\mathrm{DyCo}_{2}$ and the transition smears gradually

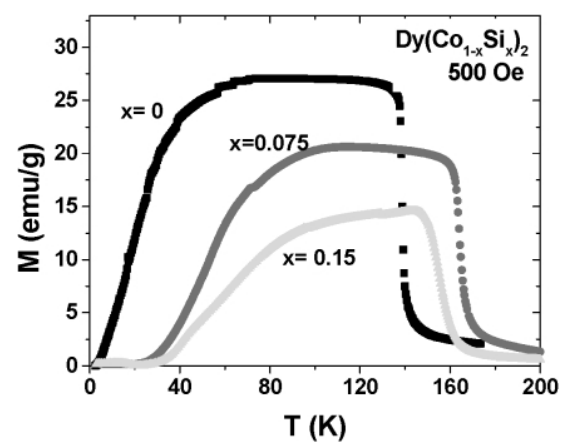

Fig. 1. Temperature variation of magnetization in $\mathrm{Dy}\left(\mathrm{Co}_{1-\mathrm{x}} \mathrm{Si}_{\mathrm{x}}\right)_{2}$ compounds at 500 Oe.
Table 1

The values of lattice parameter $(a)$, ordering temperature $\left(T_{\mathrm{C}}\right)$, saturation magnetization $\left(M_{\mathrm{S}}\right)$ and $3 \mathrm{~d}$ magnetic moment $\left(\mu_{3 \mathrm{~d}}\right)$ in $\mathrm{Dy}\left(\mathrm{Co}_{1-x} \mathrm{Si}_{x}\right)_{2}$ compounds

\begin{tabular}{lllll}
\hline$X$ & 'a' (̊) & $T_{\mathrm{C}}(\mathrm{K})$ & $M_{\mathrm{S}}\left(\mu_{B} /\right.$ f.u. $)$ & $\mu_{3 \mathrm{~d}}\left(\mu_{B} /\right.$ atom $)$ \\
\hline 0.0 & 7.179 & 139 & 7.07 & 0.96 \\
0.075 & 7.189 & 164 & 7.21 & 0.97 \\
0.15 & 7.196 & 154 & 7.18 & 1.07 \\
\hline
\end{tabular}

with $\mathrm{Si}$ concentration. It has already been reported that $\mathrm{DyCo}_{2}$ shows FOT and this is attributed to the IEM [3,5]. According to the model by Khmelevskyi and Mohn, the lattice parameter, ' $a$ ' of $\mathrm{DyCo}_{2}$ is well within the range $7.05<a<7.22 \AA$, thereby satisfying the condition for IEM. This is because of the fact that the lattice parameter has to be more than $7.22 \AA$ to enable spontaneous and stable moment formation in the Co sublattice. Therefore, Co sublattice in $\mathrm{DyCo}_{2}$ is nonmagnetic above the ordering temperature. On cooling the sample through the ordering temperature, the Co sublattice becomes magnetic due to the presence of the exchange field of Dy and this is termed as IEM. This moment formation and the subsequent ordering in the Co sublattice are responsible for the FOT. Fig. 2(a) and (b) show the Arrott plots of $\mathrm{DyCo}_{2}$ and $\mathrm{Dy}\left(\mathrm{Co}_{0.925}\right.$ $\left.\mathrm{Si}_{0.075}\right)_{2}$, respectively. The onset of IEM in $\mathrm{DyCo}_{2}$ is clearly seen in the S-shaped Arrott plots in Fig. 2(a). If the Co

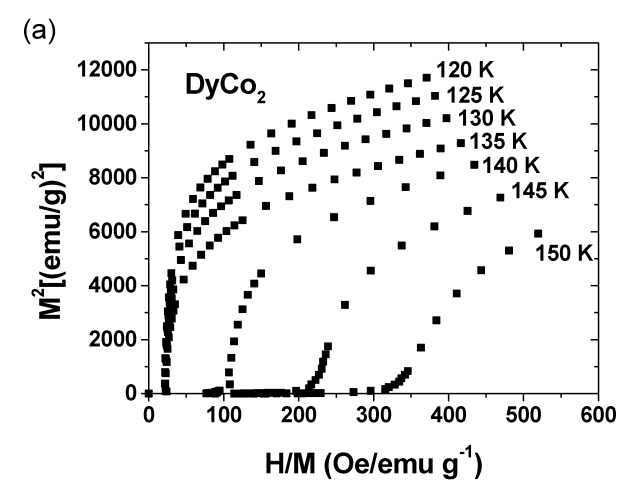

(b)

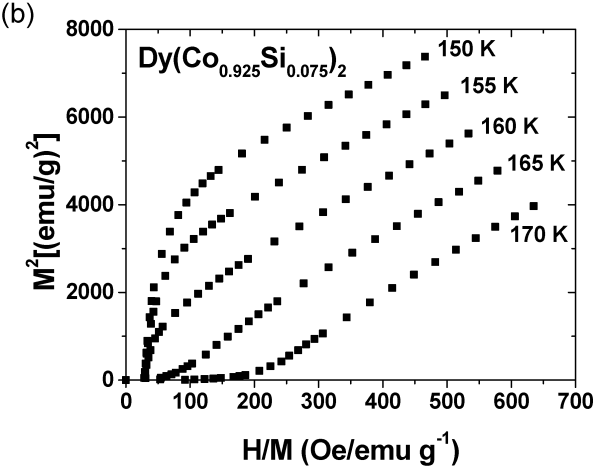

Fig. 2. Arrott plots for (a) $\mathrm{DyCo}_{2}$ and (b) $\mathrm{Dy}\left(\mathrm{Co}_{0.925} \mathrm{Si}_{0.075}\right)_{2}$. 
sublattice has a permanent moment above the ordering temperature, the Co sublattice would always be spontaneously magnetic and the transition at $T_{\mathrm{C}}$ would be of second order, as seen in many intermetallics. This is the case in $\mathrm{RCo}_{2}$ compounds containing light rare earths in which the lattice parameters are more than the critical value of $7.22 \AA$ [5]. It is interesting to see that Duc et al. [9] have reported the critical lattice parameter to be $7.27 \AA$ for the enhancement of $T_{\mathrm{C}}$ as a result of $\mathrm{Al}$ substitution in $\mathrm{R}(\mathrm{Co}, \mathrm{Al})_{2}$ compounds. The increase in $T_{\mathrm{C}}$ in this case has been attributed to the formation of moments in the Co sublattice $[9,10]$. Therefore, there is a strong correlation between the lattice parameter and $\mathrm{Co}$ magnetism in $\mathrm{RCo}_{2}$-based compounds. Recent results on MCE and MR seem to favor $7.22 \AA$ as the critical lattice parameter to observe IEM and FOT in $\mathrm{RCo}_{2}$ compounds [3].

As mentioned above, there are several experimental results in the literature that are in agreement with the predictions of Khmelevskyi and Mohn model. It has indeed been experimentally observed that $\mathrm{YCo}_{2}$ undergoes a metamagnetic transition at applied magnetic fields of about $70 \mathrm{~T}$ [7]. The appearance of Co moments and the subsequent increase of $T_{\mathrm{C}}$ in $\mathrm{Al}$-substituted $\mathrm{Y}(\mathrm{Lu}) \mathrm{Co}_{2}$ compounds followed by lattice expansion [10] also support this model. The role of lattice parameter in determining the magnetic state of $\mathrm{Co}$ is also revealed by the pressure dependence of $T_{\mathrm{C}}$ [6] and also by thermal expansion anomalies in various $\mathrm{RCo}_{2}$ compounds [8].

However, the magnetic transitions observed in $\mathrm{Si}$ substituted $\mathrm{DyCo}_{2}$ compounds in the present case are of second order, as revealed by the Arrott plots and also by the lower MCE values (discussed later). Arrott plots for these two compounds are not $\mathrm{S}$ - shaped, as can be seen from Fig. 2(b) for $\mathrm{Dy}\left(\mathrm{Co}_{0.925} \mathrm{Si}_{0.075}\right)_{2}$. Therefore, it is clear that there is no IEM in these Si-substituted compounds. It has been reported that in $\operatorname{Er}\left(\mathrm{Co}_{1-x} \mathrm{Si}_{x}\right)_{2}$ [8] and $\mathrm{Ho}\left(\mathrm{Co}_{1-x} \mathrm{Si}_{x}\right)_{2}$ [11] the transition is FOT for $x \leq 0.075$ and it changes to SOT for $x>0.075$. A similar variation has been observed in Alsubstituted $\mathrm{RCo}_{2}$ compounds $[9,12,13]$ as well. A common trend seen in both $\mathrm{Si}$ and $\mathrm{Al}$ substituted compounds is that the critical concentration (of $\mathrm{Si}$ or $\mathrm{Al}$ ) at which FOT changes to SOT is more in Er compounds and it becomes progressively smaller in Dy compounds. It is of interest to note that according to Khmelevskyi and Mohn, the magnetic transitions should be first order in Dy, Ho and Er compounds even for $x=0.15$ in both $\mathrm{Si}$ and Al substitutions, since the lattice parameter is less than the critical value. The reason for this variation can be attributed to spin fluctuations in the 3d sublattice.

Spin fluctuations in these compounds arise due to two reasons. In addition to the usual thermal contribution, the instability of moments in the region $a<a_{\mathrm{c}}$, also contributes to spin fluctuations. Increase in the lattice parameter as a result of $\mathrm{Si}$ or $\mathrm{Al}$ substitution increases the tendency for the moment formation in the $3 \mathrm{~d}$ sublattice because of the magnetovolume effect. However, it should be noted that this moment formation is only local and these moments are not strongly exchange coupled. This gives rise to a weakly ferromagnetic phase with fluctuating moments [9]. From the variation of electronic specific heat coefficient $(\gamma)$, Wada et al. [10] have established the presence of enhanced spin fluctuations in this region $\left(a<a_{\mathrm{c}}\right)$. Duc et al. [9] have also reported the presence of larger spin fluctuations in this region compared to that in the region $a>a_{\mathrm{c}}$, based on magnetic and electrical resistivity studies in $\mathrm{R}(\mathrm{Co}, \mathrm{Al})_{2}$ compounds. Since spin fluctuations suppress IEM, there is a trend towards SOT on $\mathrm{Si}$ or $\mathrm{Al}$ substitution. This is the reason for the change from FOT to SOT in $\operatorname{Er}\left(\mathrm{Co}_{1-x} \mathrm{M}_{x}\right)_{2}$ and $\mathrm{Ho}\left(\mathrm{Co}_{1-x} \mathrm{M}_{x}\right)_{2}(\mathrm{M}=\mathrm{Al}, \mathrm{Si})$ for $x>0.075$. Due to the larger lattice parameters of Dy compounds compared to that of the corresponding Ho and Er compounds, the local Co moment formation is more probable in the former case [13]. This enhances the spin fluctuations in the Co sublattice in Dy compounds and as a result, $\mathrm{Dy}\left(\mathrm{Co}_{1-x} \mathrm{M}_{x}\right)_{2}$ compounds show SOT at lower ' $x$ ' values as compared to the Er and Ho counterparts. A similar trend has been observed in the electrical resistivity of $\mathrm{R}(\mathrm{Co}, \mathrm{Al})_{2}$ compounds with $\mathrm{R}=\mathrm{Er}$, Ho and Dy [9]. A few other reports are also available in literature, which suggest the presence of strong spin fluctuations when condition for IEM is nearly satisfied $[14,15]$. Another reason favoring an early SOT in Dy compounds in the present case may be that the ordering temperatures are higher $(\sim 140 \mathrm{~K})$ than the $\operatorname{Er}(\sim 35 \mathrm{~K})$ and Ho $(\sim 75 \mathrm{~K})$ compounds. The spin fluctuation contribution is more at higher temperatures and this also contributes to SOT [6]. Khmelevskyi and Mohn have also reported that higher ordering temperatures lead to SOT, even in compounds where conditions for IEM exist, as in the case of $\mathrm{TbCo}_{2}[5]$.

Fig. 3 shows $\mathrm{M}$ vs. T plots under FC and ZFC conditions for $\mathrm{Dy}\left(\mathrm{Co}_{0.85} \mathrm{Si}_{0.15}\right)_{2}$. Similar plots were obtained for the compounds with $x=0$ and 0.075 as well. The large difference seen between the FC and ZFC magnetization curves, known as thermomagnetic irreversibility, is due to the domain wall pinning effect [16]. In intermetallic compounds possessing large anisotropy and low $T_{\mathrm{C}}$, width of the domain wall would be comparable to the lattice

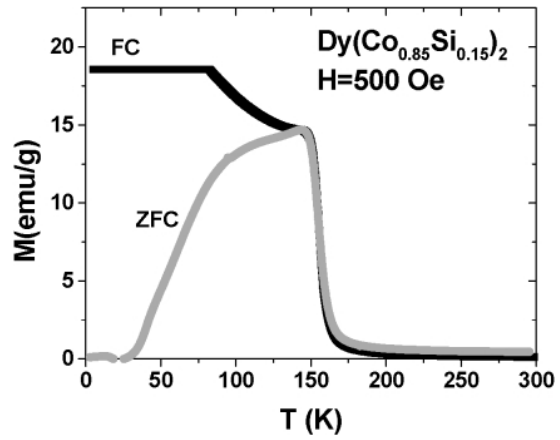

Fig. 3. Temperature variation of magnetization under FC and ZFC conditions in $\mathrm{Dy}\left(\mathrm{Co}_{0.85} \mathrm{Si}_{0.15}\right)_{2}$. 
spacing and in such systems the magnetization process is quite different as compared to that of normal ferromagnets. The most important characteristics of such narrow domain wall systems are significant FC-ZFC difference, large intrinsic coercivity and remanence at low temperatures [16]. In ZFC, at low temperatures, the domain walls are pinned and with increases of temperature the magnetization increases reaching a maximum around $T_{\mathrm{C}}$. However, in FC, the presence of the magnetic field during the cooling prevents the pinning of domain walls and therefore, the magnetization increases with the decrease in temperature. The present system, i.e. $\mathrm{Dy}\left(\mathrm{Co}_{1-x} \mathrm{Si}_{x}\right)_{2}$ can be classified as a narrow domain wall system due to the fact that the ordering temperature is much smaller (compared to $\mathrm{DyFe}_{2}$ ). Also, the anisotropy is large due to the magneto-elastic distortions taking place at the $T_{\mathrm{C}}$, as the sample is cooled from high temperatures $[17,18]$. Therefore, it is quite possible that the present system exhibits domain wall pinning effect and consequently a large thermomagnetic irreversibility. It has been reported that $\mathrm{Al}$-substituted $\mathrm{RCo}_{2}$ compounds also show similar behaviour [19].

The MCE in $\mathrm{Dy}\left(\mathrm{Co}_{1-x} \mathrm{Si}_{x}\right)_{2}$ compounds has been measured as the isothermal magnetic entropy change $\Delta S_{\mathrm{M}}(T, \Delta H)$ for various temperatures and applied magnetic fields. $\Delta S_{\mathrm{M}}$ is calculated from magnetic isotherms $M\left(T_{i}, H\right)$, obtained at a sequence of temperatures $T_{i}$, using the Maxwell's relation:

$\Delta S_{\mathrm{M}}\left(T_{\mathrm{av}, i} H_{2}\right)=\int_{H_{1}=0}^{H_{2}}\left(\frac{\partial M(T, H)}{\partial T}\right) T_{\mathrm{av}, i} \mathrm{~d} H$

$\approx \frac{1}{T_{i+1}-T_{i}} \int_{0}^{H_{2}}\left[M\left(T_{i+1}, H\right)-M\left(T_{i}, H\right)\right] \mathrm{d} H$

Here, $T_{\mathrm{av}, i}=\left(T_{i+1}+T_{i}\right) / 2$ and $\Delta T=T_{i+1}-T_{i} . \Delta S_{\mathrm{M}}$ for all the compounds for field changes $\left(\Delta H=H_{2}-H_{1}\right)$ of 1 and $4 \mathrm{~T}$ are shown in Fig. 4(a) and (b), respectively. It can be seen that $\Delta S_{\mathrm{M}}$ always shows a maximum $\left(\Delta S_{\mathrm{M}}^{\max }\right)$ close to $T_{\mathrm{C}}$ and has the highest value for $\mathrm{DyCo}_{2} . \Delta S_{\mathrm{M}}$ obtained in $\mathrm{DyCo}_{2}$ for $1 \mathrm{~T}$ in the present case compares well with the recently reported values [12,20]. For $\Delta H=4 \mathrm{~T}, \Delta S_{\mathrm{M}}^{\max }$ was $11.1 \mathrm{~J} \mathrm{Kg}^{-1} \mathrm{~K}^{-1}$ for $\mathrm{DyCo}_{2}$. The corresponding values for $\mathrm{ErCo}_{2}$ and $\mathrm{HoCo}_{2}$ are $38 \mathrm{~J} \mathrm{Kg}^{-1} \mathrm{~K}^{-1}(\Delta H=5 \mathrm{~T})$ and $22.5 \mathrm{~J} \mathrm{Kg}^{-1} \mathrm{~K}^{-1}(\Delta H=4 \mathrm{~T})$, respectively [3]. The reason for such large entropy change in these compounds is mainly the FOT at $T_{\mathrm{C}}$. In addition, the structural transitions associated with the magnetic ordering may also contribute to the MCE. Such field-induced structural transitions are known to cause large MCE in various intermetallics [2]. The relatively lower value of $\Delta S_{\mathrm{M}}^{\max }$ in $\mathrm{DyCo}_{2}$ can be attributed to the presence of spin fluctuations mentioned earlier. It is of interest to note that $\Delta S_{\mathrm{M}}^{\max }$ for $\mathrm{TbCo}_{2}$, which exhibits SOT is $6.5 \mathrm{~J} \mathrm{Kg}^{-1} \mathrm{~K}^{-1}(\Delta H=5 \mathrm{~T})$. The fact that this value is not negligible compared to that of $\mathrm{DyCo}_{2}$, suggests that the transition in $\mathrm{TbCo}_{2}$ is predominantly SOT, but has some signature of FOT. MR studies have also shown that maximum MR in $\mathrm{TbCo}_{2}$ is about $17 \%$ whereas it is about (a)
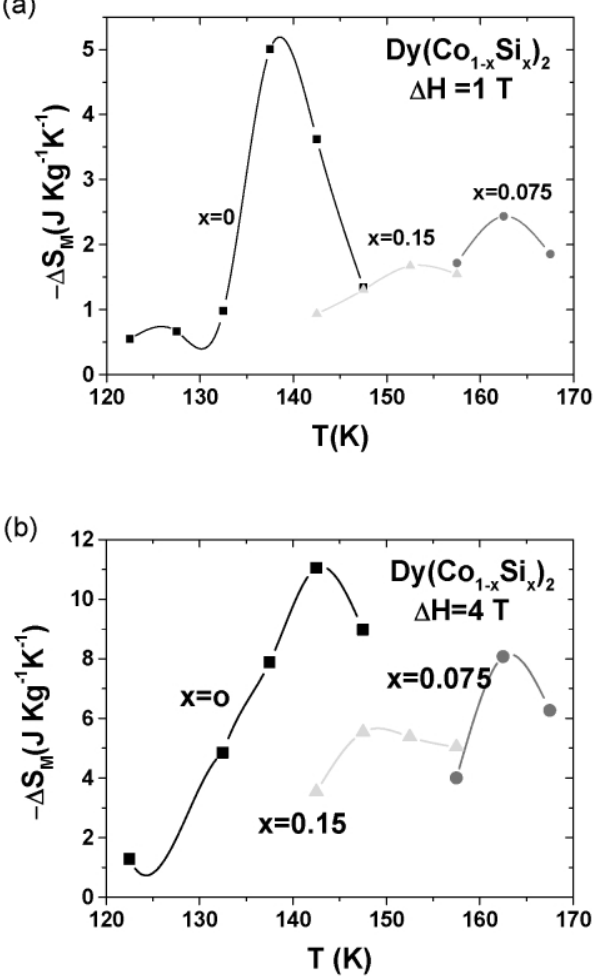

Fig. 4. Isothermal entropy change in $\mathrm{Dy}\left(\mathrm{Co}_{1-x} \mathrm{Si}_{x}\right)_{2}$ compounds for $\Delta H=1 \mathrm{~T}(\mathrm{a})$ and $4 \mathrm{~T}(\mathrm{~b})$.

$30 \%$ in $\mathrm{DyCo}_{2}$ and $60 \%$ for $\mathrm{HoCo}_{2}$ [3]. In this context, it is of importance to note that Khmelevskyi and Mohn model predicts conditions favoring IEM in $\mathrm{TbCo}_{2}$ [5]. Considerable values of MCE and MR in $\mathrm{TbCo}_{2}$, therefore, seem to support this model.

It can also be seen from Fig. 4 that $\Delta S_{\mathrm{M}}^{\max }$ decreases with $\mathrm{Si}$ substitution and this is due to the disappearance of IEM and the subsequent change in the order of transition. $\Delta S_{\mathrm{M}}^{\max }$ in $\mathrm{Dy}\left(\mathrm{Co}_{0.85} \mathrm{Si}_{0.15}\right)_{2}$ is lower than that of $\mathrm{TbCo}_{2}$ though both these compounds show SOT. This may be due to the larger contribution from the spin fluctuations in $\mathrm{Dy}\left(\mathrm{Co}_{0.85} \mathrm{Si}_{0.15}\right)_{2}$ compared to that in $\mathrm{TbCo}_{2}$. Reasonably large values of $\Delta S_{\mathrm{M}}^{\max }$ in $\mathrm{Dy}\left(\mathrm{Co}_{0.925} \mathrm{Si}_{0.075}\right)_{2}$ along with an increase in $T_{\mathrm{C}}$ of about $25 \mathrm{~K}$, as compared to $\mathrm{DyCo}_{2}$, makes this Sisubstituted system promising from the point of view of magnetic refrigeration applications around $150 \mathrm{~K}$. Furthermore, in the temperature range $80-250 \mathrm{~K}$ where adiabatic cooling is minimal, there is a growing demand for magnetic refrigerant materials possessing considerable low field $\Delta S_{\mathrm{M}}^{\max }$ [2].

\section{Conclusion}

In conclusion, IEM is found to be responsible for FOT in $\mathrm{DyCo}_{2}$-based compounds. Spin fluctuations suppress IEM, 
thereby making the transitions second order. Low temperature magnetization behaviour in these compounds is governed by domain wall pinning effect.

\section{Acknowledgements}

One of the authors (KGS) thanks the B. R. N. S. (D. A. E.), Govt. of India for the financial support in the form of a sponsored project.

\section{References}

[1] R.J. Radwanski, R. Michalski, Z. Ropka, A. Blaut, Physica B 319 (2002) 78

[2] V.K. Pecharsky, K.A. Gschneidner Jr., J. Magn. Magn. Mater. 200 (1999) 44

[3] N.H. Duc, D.T.K. Anh, P.E. Brommer, Physica B 319 (2002) 1.

[4] E.P. Wohlfarth, P. Rhodes, Phil. Mag. 7 (1962) 1817.

[5] S. Khmelevskyi, P. Mohn, J. Phys.: Condens. Matter 12 (2000) 9453.

[6] H. Yamada, K. Fukamichi, T. Goto, Phys. Rev. B 65 (2001) 24413.

[7] H. Yamada, K. Fukamichi, T. Goto, Physica B 327 (2003) 148.
[8] T.D. Cuong, N.H. Duc, P.E. Brommer, Z. Arnold, J. Kamarad, V. Sechovsky, J. Magn. Magn. Mater. 182 (1998) 143.

[9] N.H. Duc, V. Sechovsky, D.T. Hung, N.H.K. Ngan, Physica B 179 (1992) 111.

[10] H. Wada, M. Shiga, Y. Nakamura, J. Magn. Magn. Mater. 9091 (1990) 727.

[11] N.H. Duc, T.K. Oanh, J. Phys. Condens. Matter 9 (1997) 1585.

[12] H. Liu, D. Wang, S. Tang, Q. Cao, T. Tang, B. Gu, Y. Du, J. Alloys Compd. 346 (2002) 314.

[13] N.H. Duc, T.D. Hien, P.E. Brommer, J.J.M. Franse, J. Magn. Magn. Mater. 104-107 (1992) 1252.

[14] R. Hauser, E. Bauer, E. Gratz, H. Muller, M. Rotter, H. Michor, G. Hilscher, A.S. Markosyan, K. Kamishima, T. Goto, Phys. Rev. B 61 (2000) 1198.

[15] N.V. Baranov, A.N. Pirogov, J. Alloys Compd. 217 (1995) 31.

[16] J.L. Wang, C.C. Tang, G.H. Wu, Q.L. Liu, N. Tang, W.Q. Wang, W.H. Wang, F.M. Yang, J.K. Liang, F.R. de Boer, K.H.J. Buschow, Solid State Commun. 121 (2002) 615.

[17] R.Z. Levitin, A.S. Markosyan, J. Magn. Magn. Mater. 84 (1990) 247

[18] E. Gratz, A. Lindbaum, A.S. Markosyan, H. Mueller, A.Y. Sokolov, J. Phys. Condens. Matter 6 (1994) 6699.

[19] A. Kowalczyk, A. Szajek, J. Baszynski, J. Kovac, G. Chelkowska, J. Magn. Magn. Mater. 166 (1997) 237.

[20] D.H. Wang, S.L. Tang, H.D. Liu, W.L. Gao, Y.W. Du, Intermetallics 10 (2002) 819. 